\title{
Effects of Core Stabilization Exercise versus General Trunk- Strengthening Exercise on Balance Performance, Pain Intensity and Trunk Muscle Activity Patterns in Clinical Lumbar Instability Patients: A Single Blind Randomized Trial
}

\author{
Rungthip PUNTUMETAKUL ${ }^{1,2, *}$, Pongsatorn SAIKLANG ${ }^{1,2}$, \\ Wantanee YODCHAISARN ${ }^{1,3}$, Torkamol HUNSAWONG ${ }^{1,2}$ and \\ Janyawat RUANGSRI ${ }^{1,2}$
}

\author{
${ }^{1}$ Research Center in Back, Neck, Other Joint Pain and Human Performance (BNOJPH), \\ Faculty of Associated Medical Sciences, Khon Kaen University, Khon Kaen 40002, Thailand \\ ${ }^{2}$ School of Physical Therapy, Faculty of Associated Medical Sciences, Khon Kaen University, \\ Khon Kaen 40002, Thailand \\ ${ }^{3}$ School of Allied Health Sciences, Walailak University, Nakhon Si Thammarat 80160, Thailand
}

('Corresponding author's e-mail: rungthiprt@gmail.com)

Received: 20 October 2019, Revised: 14 June 2020, Accepted: 15 July 2020

\begin{abstract}
Postural balance is important for activities of daily living. Patients with chronic low back pain (CLBP) exhibit balance problems, such as difficulty performing daily activities. Specifically, CLBP patients with clinical lumbar instability (CLI) have demonstrated deep trunk muscular dysfunction that induces poor postural control and may result in a high risk of falling. This study aimed to investigate the effect of a 10-week core stabilization exercise (CSE) regimen versus a strengthening trunk exercise (STE) regimen and conduct a 3-month follow-up on balance, pain, and trunk muscle activity patterns in CLI patients. A single-blind randomized controlled trial was used to compare the effects of CSE and STE on balance, pain, and trunk muscle activity patterns in 38 CLBP patients with CLI. The results of the 5times-sit-to-stand test (FTSST) and pain intensity revealed significant improvements in both groups after ten weeks of exercise and at the 3-month follow-up point. However, the differences in the FTSST scores and pain intensity between the CSE and STE patients were not significant. Moreover, the CSE group presented significantly greater deep abdominal muscle activation than the STE group after ten weeks of exercise. In addition, both groups exhibited a trend of improvement in terms of deep back muscle activation. Therefore, the current study results suggest that both exercise regimens can improve balance performance and pain intensity. Moreover, the CSE group showed more significant improvements in deep abdominal muscle activation than the STE group.
\end{abstract}

Keywords: Clinical lumbar instability, Balance performance, Trunk muscle activity pattern, Core stabilization exercise, Strengthening exercise

\section{Introduction}

Low back pain (LBP) is the most common musculoskeletal disorder [1,2], with a world prevalence of approximately $25-84 \%[1,3,4]$. Previous studies have reported that the 1 -year prevalence of LBP in Thailand is $49-77 \%$ [5,6]. Most LBP cases are benign, which may disappear within six weeks. However, approximately $20 \%$ of individuals with LBP do not report any improvement in their condition, leading to chronic low back pain (CLBP) [7]. CLBP interferes with biomechanical changes and decreases 
http://wjst.wu.ac.th

an individual's ability to work, which leads to reduced productivity and socialization and an increased economic burden due to medical costs [8,9]. CLBP has several subcategories, one of which is clinical lumbar instability (CLI). CLI is defined as the inability of the spine to maintain its normal displacement patterns under physiologic loads, so there is no initial or additional neurologic deficit, no significant deformity, and no incapacitating pain [10]. Prior research has demonstrated that CLI accounts for $12-13$ $\%$ of CLBP cases $[11,12]$.

In CLI patients, previous studies reported that they suffer from low back pain $[13,14]$ and exhibit changes in the trunk muscle recruitment pattern [15-17]. These problems are associated with balance performance, which requires interaction between the musculoskeletal and neural control systems [18-20]. Moreover, previous research has revealed that clinical lumbar instability patients show a significant increase in path length and mean velocity in the anterior-posterior and medial-lateral directions as well as the total area of excursion when compared with healthy individuals [21]. Therefore, CLBP patients with CLI could have poor balance.

Postural balance, which is controlled by sensory information, central processing, and neuromuscular responses, is important for activities of daily living [18,19,22]. Sensory information consists of the vestibular, visual, and somatosensory (cutaneous and proprioceptive) systems [19,23]. Many researchers have reported that diminished postural control manifests as problems with balance, such as performing static and dynamic postures [22,24-26]. In CLBP, sensory information may be altered due to the trunk muscles and surrounding structures' poor quality [27,28]. Pain, and its effect on postural balance, is an important factor [29] and may lead to an increase in the presynaptic inhibition of muscle input. Moreover, pain is associated with decreased proprioception and muscle strength, affecting the sensory information and compromising the relationship between postural responses and sensory information [30]. Furthermore, CLBP is associated with experiencing multiple falls (odds ratio between 1.54 to 3.90) [3133]. Proprioception is the complex interaction between efferent and afferent fibres in order to control body movement and posture. Silfies et al. (2005) reported that patients with clinical lumbar instability demonstrated lower abdominal synergist ratios-the internal oblique and rectus abdominis as well as the external oblique and rectus abdominis-than healthy controls $(p<0.05)[34,35]$. Specifically, CLBP patients with CLI present with deep trunk muscle (transversus abdominis (TrA), internal oblique (IO), and lumbar multifidus (LM) dysfunction [10], which could impair proprioception and induce poor postural control [36].

Two muscular systems provide stability and movement for the lumbar spine: The deep and superficial muscular systems [37]. The deep muscular system consists of the TrA and IO as well as the LM muscles. The deep muscular system has been proposed to function primarily as a force transducer that provides feedback on spinal position and movements to the neural control subsystem [38,39]. The LM muscle is better suited for segmental control. The multifidus muscle is proposed to function as a stabilizer during lifting and rotational movements of the lumbar spine [38-40], whereas the TrA is thought to contribute to spinal stability by creating a rigid cylinder around the spine and increasing the stiffness of the lumbar spine [38,40]. Izzo et al. (2013) suggested that the role of the deep trunk muscles is to produce a stiffening effect on the lumbar spine through an attachment to the thoracolumbar fascia in conjunction with an increase in intra-abdominal pressure; eventually, this could result in spinal stability in patients with clinical lumbar instability $[15,38,41]$. The superficial muscle system consists of the rectus abdominis (RA) and iliocostalis lumborum pars thoracis (ICLT) muscles. As these muscles do not directly attach to the lumbar spine, they cannot influence the lumbar motion segments. These muscles act as mobilizing muscles that demonstrate non-continuous activation to produce general gross trunk stabilization and generate large torque for trunk movement as well as spinal compression [35,38]. They are also crucial in the shock absorption of the loads [42].

The strengthening trunk exercise (STE) is commonly used in LBP patients to improve pain, physical disability, and trunk muscle activity $[13,43,44]$. The STE could activate the abdominal and paraspinal muscles as a whole at a relatively high contraction level [45]. Clinical studies using the core stabilization exercise (CSE) have demonstrated pain relief and the potential to improve an individual's physical abilities, quality of life, and trunk muscle activation [13-16]. The CSE could also enhance neuromuscular control skills as well as retrain deep abdominal and back muscles around the lumbar spine. The exercises 
http://wjst.wu.ac.th

progressed when applying a low load to the muscles through controlled movements [13, 14,46]. Andrusaitis et al. (2011) compared the effects of CSE and STE in CLBP patients. They reported that CSE more effectively relieved pain and improved physical ability than STE. However, the balance did not differ between the CSE and STE groups [47]. However, Kaji et al. (2010) presented a statistically significant decrease in the centre of pressure (COP) displacement in CLBP patients after CSE training. This finding may be related to an improvement in neuromuscular control. Therefore, they proposed that CSE programs of the lumbo-pelvis-hip complex should be considered important in improving balance [48]. In their previous study, Puntumetakul et al. (2013) demonstrated that a 10-week and 3-month follow-up of CSE training could improve pain-related outcomes in chronic low back pain patients with CLI [16]. Moreover, the CSE can effectively relieve pain, which might then improve balance $[29,30,47,49]$. However, no studies have reported on the effect of 10-week and 3-month follow-up CSE on balance performance in chronic low back pain patients with CLI.

We hypothesized that CSE training specific to deep trunk muscle could significantly improve balance performance, pain intensity, and trunk muscle activity patterns than STE training. However, research comparing the effects of the CSE and STE in CLBP patients with CLI in terms of balance performance, pain intensity, and trunk muscle activity patterns is limited. Therefore, this study aimed to investigate the effect of a 10-week (and 3-month follow-up) CSE regimen versus STE training on balance performance, pain intensity, and trunk muscle activity patterns in patients with CLI.

\section{Materials and methods}

Study design

A single-blind randomized controlled trial was used to compare the effects of CSE and STE on balance performance, pain intensity, and trunk muscle activation patterns in CLBP patients with CLI. This study was conducted at the Research Center in Back, Neck, and Other Joint Pain and Human Performance (BNOJPH) Laboratory at Khon Kaen University in Thailand. Ethical approval for this research was obtained from the Human Research Ethics Committee (HE572089) of the University of Khon Kaen.

\section{Participants}

Between May 2014 and January 2016, 38 clinical lumbar instability patients were recruited to participate in this study using a convenience sampling method with the Srinagarind hospitals in the Khon Kaen province. A physical therapist and physiatrists screened all participants in terms of medical history, physical examination, and radiographs in order to exclude other spinal conditions, such as spondylolisthesis and scoliosis [10,14,15]. Clinical lumbar instability was confirmed by the Delphi checklist, in which expert practitioners defined common identifiers of lumbar clinical instability. Moreover, Cook et al. (2006) suggested that the Delphi checklist is associated explicitly with clinical lumbar instability and may be beneficial for clinical differential diagnosis [13,20]. CLI was diagnosed by the Delphi checklist using a score of 7 out of 13 in subjective aspects and a positive sign in at least 2 out of 3 of the following provocation tests: Painful catch, aberrant movement sign, or prone instability test $[13,16]$. Exclusion criteria were as follows: Any back or other joint disorders, deformities, or fracture (spine, pelvic, and lower limbs), obvious abnormal movement of the lumbar motion segment when assessing flexion-extension radiographs (i.e., sagittal plane translation larger than $4.5 \mathrm{~mm}$ of the vertebral body width according to others or sagittal plane rotation larger than 15 degrees at L1-L2, L2-L3 or L3-L4, 20 degrees at L4-L5, or 25 degrees at L5-S1) [10,15], visual problems that were not corrected with glasses or contact lenses, vestibular or brain disorders, spinal cord disorders, systemic diseases (e.g., diabetes with neuropathy) that could affect balance, and/or regularly performed CSE or STE exercises $[14,15]$. The sample size, using a formula of repeated measures analysis of variance, was calculated from the difference in the mean change in the FTSST scores between the CSE and STE patients one day after ten weeks of intervention in 10 participants $(\mathrm{CSE}=5, \mathrm{STE}=5)$ obtained from a pilot study, assuming 90 
http://wjst.wu.ac.th

$\%$ power and a $20 \%$ attrition rate. An optimal sample size per group was calculated to be at least 19 individuals.

\section{Procedure}

This study was conducted according to the flowchart in Figure 1. All participants were screened via medical history and physical examination. Two participants were excluded due to their experiencing a prominent abnormal movement of the lumbar motion segment during the flexion-extension radiographs assessment (sagittal plane translation $>4.5 \mathrm{~mm}$ ). All the participants were asked to sign a written informed consent form before participation. At baseline, balance performance, pain intensity, and muscle activation patterns were evaluated by a physical therapist with 20 years of experience (WY). Then, the participants were asked to choose an opaque, sealed envelope that would randomly assign them to either the CSE or STE group. Block randomized allocation with a block size of 2 was used in this study. A physical therapist undertook the randomization process with six years of experience (PS) who was not involved in the recruitment, treatment, or evaluation.

Both the CSE and STE exercises were supervised by a physical therapist with 30 years of experience (RP). All participants performed $220 \mathrm{~min}$ sessions of exercise per week for ten weeks. The participant did their exercise through the physical therapist by face to face. The participants had their exercise performance re-assessed every session to determine whether they correctly performed the previous exercise. The progress of the training was based on individual performance. If any participants failed to perform the previous exercise accurately, they were retrained on the previous exercise until they succeeded. The progression of both exercises is in the same condition. Both groups were required to practice the exercise daily at home using the same level, position, and repetition as in the clinic. A researcher (RP) made a telephone call to motivate all participants every week to continue their daily home exercises.

The day after the completion of the training, all participants were asked to return to the clinic for evaluation as well as again after three months by a researcher (WY) who was blinded to the experimental procedures.

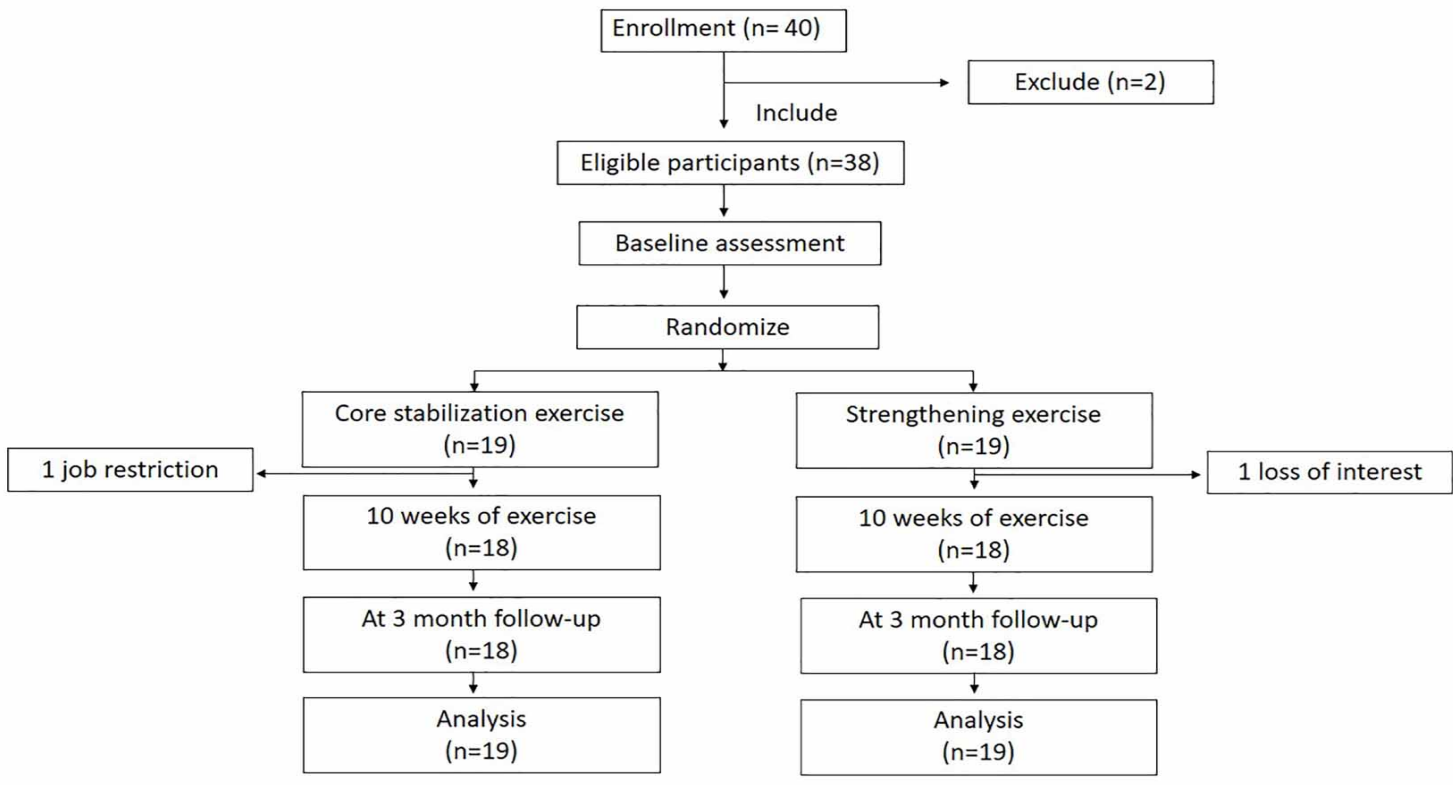

Figure 1 Flow of participants through the study. 


\section{Exercise intervention}

Core stabilization exercise

Details on the CSE are described in our previous study [16]. This program prescribed 20 $\mathrm{min} / \mathrm{session}$, two sessions/week with a physical therapist in addition to a daily home program for ten weeks. The core stabilization exercise (abdominal drawing-in maneuver) aims to improve neuromuscular control skills, help relearn normal function, and enhance the strength and endurance of the local muscle system around the lumbar spine, such as the transversus abdominis and lumbar multifidus muscles, in order to maintain static and dynamic spinal stability [50,51]. The 10-week exercise program was divided into 3 phases. The 1 st phase focused on correctly isolating the TrA and LM muscles' low-load activation using an abdominal drawing-in maneuver (ADIM) technique. A pressure biofeedback device (Chattanooga Australia Pty Ltd, Brisbane, QLD, Australia) and electromyography biofeedback (MP100, BIOPAC Systems Inc., Goleta, CA, USA) were used to facilitate correct performance during training. The 2nd phase involved the application of the low load to the muscles through controlled movements of the upper and lower extremities. The last phase aimed to integrate this co-activation into functional tasks.

\section{Strengthening exercise}

The STE program activated the erector spinae muscles and abdominis muscles through body extension and flexion, respectively. This program was adapted from a previous program [43] that extended the exercise from 8 weeks to 10 weeks. The progression of the exercise program was based on individual performance. Each set in the program was repeated 10 times and held for $10 \mathrm{~s}$, with 5 min of rest between sets.

\section{Outcome measurements}

\section{Primary outcome}

\section{Balance performance}

The 5-times-sit-to-stand test (FTSST) is a clinical balance test for investigating balance performance. The FTSST score is defined as the amount of time spent alternatively standing up from and sitting down on a chair in 5 repetitions. The starting position is a seated position on an adjustable-height chair, in which its height can be adjusted to the participant's leg length. Participants were asked to sit with half of their thighs on the seat with their hips and knees bent at approximately 90 degrees and their feet flat on the floor. The researcher provided the following instructions according to the standardized laboratory protocol [52,53]: "I want you to stand up and sit down five times as quickly as you can-but safely-when I say 'Go." Timing began when the examiner said "Go" and stopped when the subject's buttocks touched the fifth repetition chair. The participants were asked to stand up for each repetition completely. Additionally, the participants were allowed to practice two times before the test. The participants performed three trials of the FTSST with 1 min of rest between trials; the average of the scores from the three trials was collected for statistical analysis. This test has excellent inter-rater $(\mathrm{ICC}=$ 0.99) [53] and intra-rater (ICC $=0.89$ ) [52] reliability and validity and also correlates with the Timed Up and Go test (Pearson's correlation $r=0.64, p<0.001$ ) and Dynamic gait index (Spearman $\mathrm{p}=-0.68$ ) $[52,54]$. The minimal clinically important change (MCIC) for balanced performance on the FTSST is reported to be at least $5 \mathrm{~s}$ for people with chronic musculoskeletal pain [55]. To ensure the reliability of the FTSST, the intra-rater reliability was determined using 10 CLBP with CLI participants. The intrarater study was conducted with the researcher (WY). The intra-rater reliability for the FTSST measurements was analyzed using the intra-class correlation coefficient (ICC 3, 1). The results indicated an excellent intra-rater reliability of the FTSST (ICC 3,1 =0.98).

\section{Secondary outcomes}

\section{Pain intensity}

An 11-point numeric rating scale (NRS) with verbal reports at either end of the exercise was chosen for the self-reported measure of pain intensity. The participants reported the intensity of their pain on a scale ranging from 0 (indicating no pain) to 10 (indicating the most pain ever experienced) [56,57]. The participants were asked to circle the number on the NRS that best described their resting pain intensity 
within $24 \mathrm{~h}$. The minimal clinically significant change (MCIC) for pain on the NRS is reported to be at least 2.5 points for persons with chronic low back pain [58].

\section{Trunk muscle activity pattern}

Trunk muscle activity patterns were measured by surface electromyography (sEMG). After light skin abrasion and alcohol cleansing, four pairs of electrodes were placed over the right side of 4 trunk muscles: This includes transverse abdominis (TrA) and internal oblique (IO), rectus abdominis (RA), lumbar multifidus (LM), and iliocostalis lumborum pars thoracis (ICLT). The details of the electrode placement were consistent with previous studies $[15,16]$. The ground electrodes of the right RA and ICLT muscles were placed over the ribcage, and the other two ground electrodes of the right TrA and IO and LM muscles were placed over the right iliac crest. The EMG signals were sampled at $1,000 \mathrm{~Hz}$ with a gain of 1,000, $10-500 \mathrm{~Hz}$ bandwidth, and a 110-dB common-mode rejection ratio. The task, abdominal drawing-in maneuver (ADIM) with a $10 \mathrm{~s}$ hold in a 4-point kneeling position, was performed three times with a 1 min rest between trials [15]. Abdominal and back muscle activities were normalized using submaximal voluntary isometric contractions. For the abdominal muscles (TrA and IO and RA), participants raised both feet $1 \mathrm{~cm}$ from the floor in a crook lying position with a $5 \mathrm{~s}$ hold. For the back muscles (LM and ICLT), participants performed a double knee raise $5 \mathrm{~cm}$ from the floor in a prone lying position with a $5 \mathrm{~s}$ hold $[15,16]$. The raw EMG signal was first visually checked for electro cardiac artefacts. The root mean square values of the EMG signals produced during an isometric hold of these activities were then used to normalize the sEMG signals obtained during the ADIM. The abdominal (TrA and IO/RA) activation ratios and back (LM/ICLT) muscles were then calculated using the normalized data. Previous studies have not reported a value for the MCIC in trunk muscle activity patterns. For the trunk muscle activity's reliability, intra-rater reliability was determined using 10 CLBP with CLI participants. The intra-rater study was conducted with the researcher (WY), and the intra-rater reliability of the trunk muscle activity measurements was analyzed using the intra-class correlation coefficient (ICC $3,1)$. The results indicated an excellent intra-rater reliability of the sub-maximal voluntary isometric contractions of TrA and IO (ICC 3,1 = 0.98), RA (ICC 3,1 = 0.99), LM (ICC 3,1 = 0.97), and ICLT (ICC $3,1=0.97)$. In addition, the results showed an excellent intra-rater reliability of the ADIM of the TrA and IO (ICC 3,1 = 0.98), RA (ICC 3,1=0.97), LM (ICC 3,1 =0.97), and ICLT $(\operatorname{ICC} 3,1=0.98)$.

\section{Data analysis}

The results are presented as means and standard deviations. All analyses were performed based on the intention-to-treat, with the last observation carried forward. SPSS version 19 for Windows was used to analyze all the data, and the Shapiro-Wilk test was used to evaluate the normality of distribution for all the data. Differences in FTSST scores, pain intensity, and trunk muscle activity patterns within a group (baseline, after 10 weeks of intervention, and at 3 months follow-up) were assessed using a one-way repeated measure ANOVA for time effect with a Bonferroni post-hoc analysis (significant at $\mathrm{p}<0.017$; $0.05 / 3$ ). An independent t-test was used to analyze the different group effects (STE and CSE exercise) at each measurement time point (significant at $\mathrm{p}<0.05$ ).

\section{Results and discussion}

Thirty-eight patients with CLI were randomly assigned to either the CSE $(n=19)$ or STE $(n=19)$ group and were analyzed in this study. One participant in the CSE group was unable to progress to the autonomous stage of the exercise. All participants in the STE group were able to progress through the exercise, completing the 10-week of STE. Table 1 shows the baseline characteristics of the participants in both groups. No significant difference in terms of characteristics and baseline outcomes was found between the groups. 
http://wjst.wu.ac.th

Table 1 Baseline characteristics of the participants.

\begin{tabular}{lccc}
\hline \multicolumn{1}{c}{ Characteristics } & CSE $(\mathbf{n}=\mathbf{1 9})$ & STE $(\mathbf{n}=\mathbf{1 9})$ & p-value \\
\hline Female:Male & $14: 5$ & $14: 5$ & 1.00 \\
Age (years), mean \pm SD & $37.26 \pm 13.38$ & $39.10 \pm 10.91$ & 0.23 \\
Weight $(\mathrm{kg})$, mean \pm SD & $57.31 \pm 8.83$ & $58.42 \pm 9.58$ & 0.72 \\
Height $(\mathrm{cm})$, mean \pm SD & $161 \pm 7.16$ & $162 \pm 6.24$ & 0.60 \\
Body mass index $\left(\mathrm{kg} / \mathrm{m}^{2}\right)$, mean $\pm \mathrm{SD}$ & $22.07 \pm 2.94$ & $22.02 \pm 2.71$ & 0.58 \\
Pain intensity (NPRS), mean $\pm \mathrm{SD}$ & $5.78 \pm 1.51$ & $5.63 \pm 1.42$ & 0.81 \\
Pain duration (months), mean $\pm \mathrm{SD}$ & $10.79 \pm 5.51$ & $10.00 \pm 5.32$ & 0.70 \\
\hline
\end{tabular}

Abbreviation: SD; standard deviation, CSE; core stabilization exercise, STE; strengthening exercise, NRS; numeric pain rating scale, kg; kilogram, $\mathrm{cm}$; centimeter.

\section{Balance performance}

The CSE group significantly improved in balance performance measured by FTSST scores from baseline to 10 weeks of exercise $(8.39 \pm 1.67$ vs. $6.71 \pm 1.10 ; p=0.001)$ as well as at the 3 -month followup $(8.39 \pm 1.67$ vs. $6.90 \pm 1.42 ; p=0.002)$ (see Table 2$)$. The STE group significantly improved in balance performance measured from baseline to 10 weeks of exercise $(9.02 \pm 1.79$ vs. $7.33 \pm 1.43 ; \mathrm{p}=$ $0.001)$ and at the 3 -month follow-up $(9.02 \pm 1.79$ vs. $7.67 \pm 1.56 ; p=0.001)$. However, no significant differences were found between the exercise groups at any measurement point.

\section{Pain intensity}

The CSE group significantly improved in pain from baseline to 10 weeks of exercise $(5.84 \pm 1.30$ vs. $1.63 \pm 1.46 ; p=0.001)$ as well as at the 3 -month follow-up $(5.84 \pm 1.30$ vs. $1.74 \pm 1.73 ; p=0.001)$. The STE group significantly improved in pain from baseline to 10 weeks of exercise $(5.74 \pm 1.56 \mathrm{vs} .2 .53$ $\pm 1.54 ; \mathrm{p}=0.001)$ and at the 3 -month follow-up $(5.74 \pm 1.56$ vs. $2.68 \pm 1.92 ; \mathrm{p}=0.001)$. However, no significant differences were found between the exercise groups.

\section{Ratio of abdominal muscles}

The TrA and IO/RA ratio of the CSE group reported a trend of improvement from baseline to 10 weeks of exercise and at the 3-month follow-up point, whereas they slightly decreased from baseline to 10 weeks of exercise and at the 3-month follow-up point in the STE group. Moreover, the TrA and IO/RA ratio of the CSE group significantly improved when compared with the STE at 10 weeks of exercise (1.18 \pm 1.89 vs. $0.14 \pm 0.14 ; p=0.001)$. However, there was no difference between both groups at the 3 -month follow-up point.

\section{Ratio of back muscles}

There was no significant improvement in the LM/ICLT ratio within and between both groups after ten weeks of exercise nor at the 3-month follow-up point. However, the back muscle ratio revealed more significant improvement in the CSE group than in the STE group for all the measurement time points. 
http://wjst.wu.ac.th

Table 2 Comparison between core stabilization exercise $(n=19)$ and strengthening exercise $(n=19)$ on FTSST, pain intensity, ratio of abdominal muscles, and ratio of back muscles.

\begin{tabular}{lccc}
\hline \multicolumn{1}{c}{ Variables } & $\begin{array}{c}\text { CSE group, } \\
\text { Mean } \pm \text { SD }\end{array}$ & $\begin{array}{c}\text { STE group, } \\
\text { Mean } \pm \text { SD }\end{array}$ & $\begin{array}{c}\text { CSE versus STE, } \\
\text { Mean difference } \pm \text { SD (95 \% CI) }\end{array}$ \\
\hline FTSST, sec & & & \\
Baseline & $8.39 \pm 1.67$ & $9.02 \pm 1.79$ & $-0.63 \pm 0.56(-1.77$ to 0.51$)$ \\
10 weeks of intervention & $6.71 \pm 1.10^{\mathrm{A}}$ & $7.33 \pm 1.43^{\mathrm{A}}$ & $-0.62 \pm 0.41(-1.47$ to 0.21$)$ \\
3-month follow-up & $6.90 \pm 1.42^{\mathrm{A}}$ & $7.67 \pm 1.56^{\mathrm{A}}$ & $-0.77 \pm 0.48(-1.75$ to 0.21$)$ \\
Pain intensity, score & & & \\
Baseline & $5.84 \pm 1.30$ & $5.74 \pm 1.56$ & $0.10 \pm 0.47(-0.84$ to 1.05$)$ \\
10 weeks of intervention & $1.63 \pm 1.46^{\mathrm{A}}$ & $2.53 \pm 1.54 \mathrm{~A}$ & $-0.90 \pm 0.49(-1.88$ to 0.09$)$ \\
3-month follow-up & $1.74 \pm 1.73^{\mathrm{A}}$ & $2.68 \pm 1.92^{\mathrm{A}}$ & $-0.94 \pm 0.59(-2.15$ to 0.25$)$ \\
Ratio of Abdominal muscles & & & \\
(TrA \& IO/RA) & & $0.94 \pm 3.31$ & $-0.66 \pm 0.76(-2.20$ to 0.88$)$ \\
Baseline & $0.28 \pm 0.20$ & $0.14 \pm 0.14$ & $1.04 \pm 0.44(0.15$ to 1.92$)$ \\
10 weeks of intervention & $1.18 \pm 1.89$ & $0.29 \pm 0.45$ & $0.03 \pm 0.12(-0.20$ to 0.27$)$ \\
3-month follow-up & $0.32 \pm 0.24$ & & \\
Ratio of Back muscles & & $1.55 \pm 0.79$ & $0.20 \pm 0.35(-0.51$ to 0.91$)$ \\
(LM/ICLT) & $1.75 \pm 1.30$ & $1.86 \pm 1.38$ & $1.04 \pm 0.54(-0.06$ to 2.14) \\
Baseline & $2.90 \pm 1.93$ & $2.17 \pm 1.62$ & $1.22 \pm 0.75(-0.31$ to 2.74) \\
10 weeks of intervention & $3.39 \pm 2.85$ & & \\
3-month follow-up & & & \\
\hline Nots: A signficat & & & \\
\hline
\end{tabular}

Notes: $\mathrm{A}=$ significant difference from baseline; within group $\mathrm{p}<0.01$ $\mathrm{B}=$ significant difference between group $\mathrm{P}=0.001$.

Abbreviation: SD; standard deviation, CSE; core stabilization exercise, STE; strengthening trunk exercise.

\section{Discussion}

The results of the current study demonstrated that the clinical balance performance and pain intensity of CLBP patients with CLI improved significantly after practicing the CSE or STE after ten weeks as well as at three months follow-up. Moreover, the CSE group's $\operatorname{TrA}$ and IO/RA ratio significantly improved compared with the STE group after ten weeks of exercise.

CLI patients suffer from pain, altered trunk muscle patterns, and poor trunk movement control [1317,21]. Poor balance in CLI patients is due to postural control requiring interaction between the musculoskeletal and nervous systems $[18,19,21]$. In terms of musculoskeletal components, patients with CLI often demonstrate an excessive range of motion (ROM) and decreased trunk muscle activation. Moreover, in terms of neural components, such patients suffer from pain during transitional activities as well as chronic pain $[16,20,21]$.

In the current study, the abdominal muscles' ratio ( $\operatorname{TrA}$ and IO/RA) significantly improved in the CSE group compared with the STE group after ten weeks of exercise $(p=0.001)$. The CSE group showed an improvement in the TrA and IO/RA ratio. It may have been due to a specific training effect of the CSE, which focuses on the preferential retraining of the TrA muscle. The results supported previous studies that the CSE improves deep abdominal muscle activation [16,59]. Puntumetakul et al. (2013) demonstrated that 10-week CSE improves pain-related outcomes and more significantly induces TrA activation in patients with CLI than stretching exercises with a hot pack [16]. Moreover, this study showed an increased ratio in the abdominal muscles following the exercise program in the CSE group, which supports the CSE's effectiveness in improving deep abdominal muscle function. Conversely, the TrA and IO/RA ratio activation slightly decreased in the STE group. The reduction of the abdominal muscles ratio ( $\operatorname{TrA}$ and IO/RA) in the STE group was probably due to the focus on the superficial abdominal muscle (RA) in the STE. This result was in agreement with the study by Atkins et al. (2015), who applied a static bracing exercise of the trunk associated with the rectus abdominis, external oblique, and erector spinae muscle in swimmers and found greater peak amplitudes of the rectus abdominis muscle via sEMG [60]. However, the ratio of abdominal muscles (TrA and IO/RA) was no different between 
http://wjst.wu.ac.th

both groups at the 3-month follow-up point. Therefore, the effect of core stabilization exercise on deep abdominal muscles cannot produce retention effects on a 3-month follow-up. The ratio of the back muscles (LM/ICLT) showed a trend of improvement in the CSE group and STE group after ten weeks of exercise as well as at the 3-month follow-up point. Therefore, the LM/ICLT ratio showed no difference between the groups in the current study.

The reduction of pain intensity might be related to deep abdominal muscle. This is aligned with previous research that reported reduced low back pain was associated with an increased TrA contraction ratio [61]. Moreover, previous research has reported that reduced low back pain is associated with an increased TrA contraction ratio [61]. Moreover, previous studies have suggested that an increase in the activation of the deep abdominal or deep back muscles may reduce the lower back's pain intensity [16, 62]. Therefore, the current study reported that the CSE group's pain intensity reported a greater trend of improvement than the STE group. This study reported that both exercise groups showed a significant improvement in low back pain after ten weeks of training and three months of follow-up. Moreover, this finding can improve low back pain scores more greatly than a clinically significant difference. According to Ostelo and de Vet (2005), the minimum clinically important difference (MCID) for the numeric rating scale (NRS) is 2.5 [58].

In addition, pain is associated with balance performance. Leveille et al. (2009) demonstrated chronic musculoskeletal pain is associated with increased falling, and more severe pain is associated with significantly higher fall rates (adjusted relative risk 1.53) [63]. Pain may lead to increased presynaptic inhibition of muscle input. An adaptation of the cortical processing of proprioceptive information can occur in CLBP [26,64]. Proprioceptive alteration is one of the possible causes of changes in postural balance in CLBP patients. Pain is associated with decreased proprioception and muscle strength, affecting sensory information and compromising the relationship between postural responses and sensory information $[30,49,65]$. Therefore, decreased pain improves clinical balance performance. In our study, both groups significantly improved in terms of balance performance as measured by FTSST scores from baseline to 10 weeks of exercise and at the 3-month follow-up point. However, this finding should be treated with some caution, as a difference in FTSST scores, while statistically significant, is not considered a clinically significant difference. According to Benaim et al. (2019), the minimum clinically important difference (MCID) for the FTSST is $5 \mathrm{~s}$ [55].

The FTSST scores and pain intensity of the CSE and STE patients were not significantly different. However, the abdominal muscles' ratio significantly improved in the CSE group after ten weeks of exercise compared with the STE group. Therefore, both exercises could improve balance performance and pain intensity in CLBP patients with CLI. However, the CSE group could more greatly improve their deep abdominal muscle activation than the STE group. Thus, we suggest that therapies should be chosen according to which exercise is the most appropriate for the problems presented by each individual patient.

A limitation of the current study is that the investigation involved CLBP patients with CLI. Therefore, the results might not apply to other groups with low back pain and CLI, such as acute and subacute low back pain. Further research is required to study the effects of CSE and STE on the balance performance of other groups with low back pain and CLI. Since the current study was limited to the investigation of back proprioception in CLBP patients with CLI, future studies should investigate the effect of the CSE and STE on back proprioception in CLBP patients with CLI.

\section{Conclusions}

The results revealed significant differences in the FTSST scores and pain intensity between the CSE and STE programs after ten weeks of exercise and at the 3-month follow-up point. However, the abdominal muscles' ratio significantly improved in the CSE group after ten weeks of exercise compared with the STE group. Therefore, this study suggests that both exercises can improve balance performance and pain intensity in chronic low back pain patients with clinical lumbar instability. Moreover, the CSE group showed greater improvements in deep abdominal muscle activation than the STE group. 
http://wjst.wu.ac.th

\section{Acknowledgements}

This study was supported and funded by the Research Center in Back, Neck, Other Joint Pain and Human Performance (BNOJPH), Khon Kaen University, Thailand.

\section{References}

[1] F Balagué, AF Mannion, F Pellisé and C Cedraschi. Non-specific low back pain. Lancet 2012; 379, 482-91.

[2] M Allegri, S Montella, F Salici, A Valente, M Marchesini, C Compagnone, B Marco, EM Maria and $\mathrm{F}$ Guido. Mechanisms of low back pain: A guide for diagnosis and therapy. F1000Research $2016 ; 5,1530$.

[3] RD Meucci, AG Fassa and NM Faria. Prevalence of chronic low back pain: Systematic review. Rev. Saude. Publica. 2015; 49, 73.

[4] SHV Oostroml, WMM Verschurenl, HCWD Vetl and HSJ Picavetl. Ten year course of low back pain in an adult population-based cohort-The Doetinchem Cohort Study. Eur. J. Pain. 2011; 15, 993-8.

[5] R Puntumetakul, W Siritaratiwat, Y Boonprakob, W Eungpinichpong and M Puntumetakul. Prevalence of musculoskeletal disorders in farmers: Case study in Sila, Muang Khon Kaen, Khon Kaen province. J. Med. Technol. Phys. Ther. 2011; 23, 297-303.

[6] P Taechasubamorn, T Nopkesorn and S Pannarunothai. Prevalence of low back pain among rice farmers in a rural community in Thailand. J. Med. Assoc. Thai. Chotmaihet. Thangphaet. 2011; 94, 616-21.

[7] R Chou. Low back pain (chronic). BMJ Clin. Evid. 2010; 2010, 1116.

[8] C Demoulin, V Distrée, M Tomasella, JM Crielaard and M Vanderthommen. Lumbar functional instability: A critical appraisal of the literature. Ann. Readapt. Med. Phys. 2007; 50, 669-84.

[9] LOP Costa, CG Maher, J Latimer, PW Hodges, RD Herbert, KM Refshauge, JH McAuley and MD Jennings. Motor control exercise for chronic low back pain: A randomized placebo-controlled trial. Phys. Ther. 2009; 89, 1275-86.

[10] MM Panjabi. Clinical spinal instability and low back pain. J. Electromyogr. Kinesiol. Off. J. Int. Soc. Electrophysiol. Kinesiol. 2003; 13, 371-9.

[11] JH Abbott, B McCane, P Herbison, G Moginie, C Chapple and T Hogarty. Lumbar segmental instability: A criterion-related validity study of manual therapy assessment. BMC Musculoskelet. Disord. 2005; 6, 56.

[12] R Puntumetakul, W Yodchaisarn, A Emasithi, P Keawduangdee, U Chatchawan and J Yamauchi. Prevalence and individual risk factors associated with clinical lumbar instability in rice farmers with low back pain. Patient Prefer. Adherence 2015; 9, 1-7.

[13] SP Kumar. Efficacy of segmental stabilization exercise for lumbar segmental instability in patients with mechanical low back pain: A randomized placebo controlled crossover study. North Am. J. Med. Sci. 2011; 3, 456-61.

[14] Y Javadian, H Behtash, M Akbari, M Taghipour-Darzi and H Zekavat. The effects of stabilizing exercises on pain and disability of patients with lumbar segmental instability. J. Back Musculoskelet Rehabil. 2012; 25, 149-55.

[15] P Areeudomwong, R Puntumetakul, K Jirarattanaphochai, S Wanpen, J Kanpittaya, U Chatchawan and J Yamauchi. Core stabilization exercise improves pain intensity, functional disability and trunk muscle activity of patients with clinical lumbar instability: A pilot randomized controlled study. $J$. Phys. Ther. Sci. 2012; 24, 1007-12.

[16] R Puntumetakul, P Areeudomwong, A Emasithi and J Yamauchi. Effect of 10-week core stabilization exercise training and detraining on pain-related outcomes in patients with clinical lumbar instability. Patient Prefer. Adherence 2013; 7, 1189-99. 
http://wjst.wu.ac.th

[17] S Davarian, N Maroufi, I Ebrahimi, F Farahmand and M Parnianpour. Trunk muscles strength and endurance in chronic low back pain patients with and without clinical instability. J. Back Musculoskelet 2012; 25, 123-9.

[18] JK Rankin, MH Woollacott, A Shumway-Cook and LA Brown. Cognitive Influence on Postural Stability: A neuromuscular analysis in young and older adults. Comp. Stud. 2000; 55, M112-M119.

[19] Y Ivanenko and VS Gurfinkel. Human postural control. Front. Neurosci. 2018; 12, 171.

[20] C Cook, J-M Brismée and Jr PS Sizer. Subjective and objective descriptors of clinical lumbar spine instability: A Delphi study. Man. Ther. 2006; 11, 11-21.

[21] W Yodchaisarn, R Puntumetakul, A Emasithi, R Boucaut and U Chatchawan. Altered postural sway during quiet standing in women with clinical lumbar instability. J. Phys. Ther. Sci. 2018; 30, 1099102.

[22] R della Volpe, T Popa, F Ginanneschi, R Spidalieri, R Mazzocchio and A Rossi. Changes in coordination of postural control during dynamic stance in chronic low back pain patients. Gait Posture 2006; 24, 349-55.

[23] RL Carvalho and GL Almeida. Assessment of postural adjustments in persons with intellectual disability during balance on the seesaw. J. Intellect. Disabil. Res. 2009; 53, 389-95.

[24] SM Henry, JR Hitt, SL Jones and JY Bunn. Decreased limits of stability in response to postural perturbations in subjects with low back pain. Clin. Biomech. 2006; 21, 881-92.

[25] S Brumagne, L Janssens, E Janssens and L Goddyn. Altered postural control in anticipation of postural instability in persons with recurrent low back pain. Gait Posture 2008; 28, 657-62.

[26] AB Braga, AC de MA Rodrigues, GVMP de Lima, LR de Melo, AR de Carvalho and GRF Bertolini. Comparison of static postural balance between healthy subjects and those with low back pain. Acta Ortop. Bras. 2012; 20, 210-2.

[27] PW Marshell and BA Murphy. Delayed abdominal muscle onsets and self-report measures of pain and disability in chronic low back pain. J. Electromyogr. Kinesiol. 2010; 20. 833-9.

[28] SP Silfies, R Mehta, SS Smith and AR Karduna. Differences in feedforward trunk muscle activity in subgroups of patients with mechanical low back pain. Arch. Phys. Med. Rehabil. 2009; 90, 1159-69.

[29] A Ruhe, R Fejer and B Walker. Center of pressure excursion as a measure of balance performance in patients with non-specific low back pain compared to healthy controls: A systematic review of the literature. Eur. Spine. J. 2011; 20. 358-68.

[30] N Karimi, I Ebrahimi, S Kahrizi and G Torkaman. Evaluation of postural balance using the biodex balance system in subjects with and without low back pain. Pak. J. Med. Sci. 2008; 24, 372-7.

[31] M Morris, D Osborne, K Hill, H Kendig, B Lundgren-Lindquist, C Browning and J Reid. Predisposing factors for occasional and multiple falls in older Australians who live at home. Aust. J. Physiother. 2004; 50, 153-9.

[32] SG Leveille, RN Jones, DK Kiely, JM Hausdorff, RH Shmerling, JM Guralnik, DP Kiel, LA Lipsitz and JF Bean. Chronic Musculoskeletal Pain and the Occurrence of falls in an older population. $J$. Am. Med. Assoc. 2009; 302, 2214-21.

[33] S Muraki, T Akune, H Oka, Y En-yo, M Yoshida, K Nakamura, H Kawaguchi and N Yoshimura. Prevalence of falls and the association with knee osteoarthritis and lumbar spondylosis as well as knee and lower back pain in Japanese men and women. Arthritis Care Res. 2011; 63, 1425-31.

[34] SP Silfies, D Squillante, P Maurer, S Westcott and AR Karduna. Trunk muscle recruitment patterns in specific chronic low back pain populations. Clin. Biomech. 2005; 20. 465-73.

[35] DA MacDonald, G Lorimer Moseley and PW Hodges. The lumbar multifidus: Does the evidence support clinical beliefs? Man. Ther. 2006; 11, 254-63.

[36] S Brumagne, L Janssens, E Janssens and L Goddyn. Altered postural control in anticipation of postural instability in persons with recurrent low back pain. Gait Posture 2008; 28, 657-62.

[37] A Bergmark. Stability of the lumbar spine. A study in mechanical engineering. Acta Orthop. Scand. Suppl. 1989; 230, 1-54.

[38] R Izzo, G Guarnieri, G Guglielmi and M Muto. Biomechanics of the spine. Part I: Spinal stability. Eur. J. Radiol. 2013; 82, 118-26. 
http://wjst.wu.ac.th

[39] PB O’Sullivan, A Burnett, AN Floyd, K Gadsdon, J Logiudice, D Miller and H Quirke. Lumbar repositioning deficit in a specific low back pain population. Spine 2003; 28, 1074-9.

[40] JKF Ng, CA Richardson, M Parnianpour and V Kippers. EMG activity of trunk muscles and torque output during isometric axial rotation exertion: A comparison between back pain patients and matched controls. J. Orthop. Res. 2002; 20, 112-21.

[41] A Bjerkefors, MM Ekblom, K Josefsson, A Thorstensson. Deep and superficial abdominal muscle activation during trunk stabilization exercises with and without instruction to hollow. Man Ther. 2010; 15, 502-7.

[42] MJ Comeford and SI Mottram. Functional stability re-training: Principles and strategies for managing mechanical dysfunction. Man Ther. 2001; 6, 3-14.

[43] GA Koumantakis, PJ Watson and JA Oldham. Trunk muscle stabilization training plus general exercise versus general exercise only: Randomized controlled trial of patients with recurrent low back pain. Phys. Ther. 2005; 85, 209-25.

[44] CA Richardson and GA Jull. Muscle control-pain control. What exercises would you prescribe? Man Ther. 1995; 1, 2-10.

[45] GA Koumantakis, PJ Watson and JA Oldham. Supplementation of general endurance exercise with stabilisation training versus general exercise only. Physiological and functional outcomes of a randomised controlled trial of patients with recurrent low back pain. Clin. Biomech. Bristol. Avon. 2005; 20, 474-82.

[46] PW Hodges and CA Richardson. Inefficient Muscular Stabilization of the lumbar spine associated with low back pain. Spine. 1996; 21, 2640-50.

[47] SF Andrusaitis, GC Brech, G FallerVitale and JMDA Greve. Trunk stabilization among women with chronic lower back pain: A randomized, controlled, and blinded pilot study. Clinics 2011; 66, 164550 .

[48] A Kaji, S Sasagawa, T Kubo and H Kanehisa. Transient effect of core stability exercises on postural sway during quiet standing. J. Strength. Cond. Res. Natl. Strength. Cond. Assoc. 2010; 24, 382-8.

[49] A Ruhe, R Fejer and B Walker. Is there a relationship between pain intensity and postural sway in patients with non-specific low back pain? BMC Musculoskelet Disord. 2011; 12, 162.

[50] KC Bliven and BE Anderson. Core stability training for injury prevention. Sports Health 2013; 5, 514-22.

[51] PW Hodges. Core stability exercise in chronic low back pain. Orthop. Clin. North Am. 2003; 34, 245-54.

[52] SL Whitney, DM Wrisley, GF Marchetti, MA Gee, MS Redfern, JM Furman. Clinical measurement of sit-to-stand performance in people with balance disorders: validity of data for the five-times-sitto-stand Test. Phys. Ther. 2005; 85, 1034-45.

[53] MJ Simmonds, SL Olson, S Jones, T Hussein, CE Lee, D Novy and H Radwan. Psychometric characteristics and clinical usefulness of physical performance tests in patients with low back pain. Spine 1998; 23, 2412-21.

[54] A Goldberg, M Chavis, J Watkins and $\mathrm{T}$ Wilson. The five-times-sit-to-stand test: Validity, reliability and detectable change in older females. Aging Clin. Exp. Res. 2012; 24, 339-44.

[55] C Benaim, S Blaser, B Léger, P Vuistiner and F Luthi. "Minimal clinically important difference" estimates of 6 commonly-used performance tests in patients with chronic musculoskeletal pain completing a work-related multidisciplinary rehabilitation program. BMC Musculoskelet Disord. 2019; 20, 16.

[56] AF Mannion, F Balagué, F Pellisé and C Cedraschi. Pain measurement in patients with low back pain. Nat. Clin. Pract. Rheumatol. 2007; 3, 610-8.

[57] A Williamson and B Hoggart. Pain: A review of three commonly used pain rating scales. J. Clin. Nurs. 2005; 14, 798-804.

[58] RW Ostelo and HCD Vet. Clinically important outcomes in low back pain. Best Pract. Res. Clin. Rheumatol. 2005; 19, 593-607.

[59] CW Hoppes, AD Sperier, CF Hopkins, BD Griffiths, MF Principe, BL Schnall, JC Bell and SL Koppenhaver. The efficacy of an eight-week core stabilization program on core muscle function and endurance: a randomized trial. Int. J. Sports Phys. Ther. 2016; 11, 507-19. 
http://wjst.wu.ac.th

[60] SJ Atkins, I Bentley, D Brooks, MP Burrows, HT Hurst and JK Sinclair. Electromyographic response of global abdominal stabilizers in response to stable- and unstable-base isometric exercise. J. Strength Cond. Res. 2015; 29, 1609-15.

[61] O Vasseljen and AM Fladmark. Abdominal muscle contraction thickness and function after specific and general exercises: A randomized controlled trial in chronic low back pain patients. Man Ther. 2010; 15, 482-9.

[62] PB O'sullivan, L Twomey and GT Allison. Altered abdominal muscle recruitment in patients with chronic back pain following a specific exercise intervention. J. Orthop. Sports Phys. Ther. 1998; 27, 114-24.

[63] SG Leveille, RN Jones, DK Kiely, JM Hausdorff, RH Shmerling, JM Guralnik, DP Kiel, LA Lipsitz and JF Bean. Chronic musculoskeletal pain and the occurrence of falls in an older population. $J$. Am. Med. Assoc. 2009; 302, 2214-21.

[64] L Mann, JF Kleinpaul, AR Pereira Moro, CB Mota and FP Carpes. Effect of low back pain on postural stability in younger women: Influence of visual deprivation. J. Bodyw. Mov. Ther. 2010; 14, 361-6.

[65] R Puntumetakul, R Chalermsan, SS Hlaing, W Tapanya, P Saiklang and R Boucaut. The effect of core stabilization exercise on lumbar joint position sense in patients with subacute non-specific low back pain: A randomized controlled trial. J. Phys. Ther. Sci. 2018; 30, 1390-5. 